José A. M. Borghans

Rob J. de Boer
Authors' address

José A. M. Borghans ${ }^{1,2}$, Rob J. de Boer ${ }^{1}$

${ }^{1}$ Theoretical Biology, Utrecht University,

Utrecht, The Netherlands.

${ }^{2}$ Department of Immunology, University Medical

Center Utrecht, Utrecht, The Netherlands.

Correspondence to:

José A. M. Borghans

Department of Immunology

KC02.085.2

University Medical Center Utrecht

Lundlaan 6

3584 EA Utrecht, The Netherlands

Tel.: +31-30-2504275

Fax: +31-30-2504305

E-mail: j.borghans@umcutrecht.nl

Acknowledgements

The authors would like to thank Becca Asquith and Ruy Ribeiro for their invaluable input in extensive discussions on the interpretation of labeling data, and are grateful to Frank Miedema and Kiki Tesselaar for critical reading of the manuscript.

\section{Quantification of T-cell dynamics: from telomeres to DNA labeling}

Summary: Immunology has traditionally been a qualitative science describing the cellular and molecular components of the immune system and their functions. Only quite recently have new experimental techniques paved the way for a more quantitative approach of immunology. Lymphocyte telomere lengths have been measured to get insights into the proliferation rate of different lymphocyte subsets, T-cell receptor excision circles have been used to quantify the daily output of new $\mathrm{T}$ cells from the thymus, and bromodeoxyuridine and stable isotope labeling have been applied to measure proliferation and death rates of naive and memory lymphocytes. A common problem of the above techniques is the translation of the resulting data into relevant parameters, such as the typical division and death rate of the different lymphocyte populations. Theoretical immunology has contributed significantly to the interpretation of such quantitative experimental data, thereby resolving diverse controversies and, most importantly, has suggested novel experiments, allowing for more conclusive and quantitative interpretations. In this article, we review a variety of different models that have been used to interpret data on lymphocyte kinetics in healthy human subjects and discuss their contributions and limitations.

Keywords: T-cell dynamics, telomeres, TREC, BrdU, stable isotopes, mathematical modeling

\section{Introduction}

Despite great advances in immunological research during the past decades, relatively little is known about the quantitative characteristics of lymphocyte kinetics. There is a large controversy on the production rates, division rates, and lifespans of mouse and human lymphocyte populations. As a consequence, fundamental questions like the maintenance of memory, the maintenance of a diverse naive lymphocyte repertoire, and the nature of homeostatic mechanisms remain largely unresolved. Many important questions in immunology are of a quantitative nature. For example, researchers are trying to show how human diseases, like HIV infection and rheumatoid arthritis, and therapeutic interventions, such as chemotherapy or hematopoietic stem cell transplantation, affect lymphocyte kinetics, but as long as there is controversy about the lymphocyte kinetics in healthy individuals, such questions remain hard to address.
Vol. 21 6: 35-47

Printed in Singapore. All rights reserved

(C) 2007 The Authors Journal compilation (C) 2007 Blackwell Munksgaard Immunological Reviews

0105-2896 
Recently, several experimental techniques have been developed that have enabled the generation of quantitative data on lymphocyte dynamics. Some are based on the quantification of natural characteristics of lymphocytes that depend on lymphocyte kinetics, such as lymphocyte telomere lengths and T-cell receptor excision circles (TRECs). Others have made use of different lymphocyte-labeling techniques, such as the fluorescent dye, carboxyfluorescein diacetate succinimidyl ester, the base analog 5-bromo-2' -deoxyuridine (BrdU), or deuterated glucose or water. Remarkably, a simple PubMed search showed that more than $10 \%$ of the recent articles in the immunological literature involve such labeling experiments (P. Hodgkin, personal communication). Yet, the interpretation of such kinetic data has turned out to be notoriously difficult (1-15). In this article, we review how mathematical models have given insights into the possibilities and limitations of the different experimental techniques and have thereby helped the quantitative interpretation of immunological data.

\section{Estimating lymphocyte turnover from telomere data}

In the 1980s, it was shown that the unique structures at the end of chromosomes consisting of tandem DNA repeats shorten with each cell division and thereby provide a record of a cell's proliferation history $(16,17)$. Because of the incapability of DNA polymerases to copy the very ends of chromosomes, each cell division leads to the loss of 50-100 terminal nucleotides from the chromosomes. This telomere shortening was shown to have functional consequences, as it limits the replication capacity of cells (18-20), and the implications of this senescence on immune responses has been addressed by mathematical modeling (21).

Weng et al. (22) made use of this phenomenon to study the replication behavior of naive and memory $\mathrm{T}$ lymphocytes. The average telomere lengths of naive and memory $\mathrm{CD} 4^{+} \mathrm{T}$ cells were found to decrease with age at very similar rates, which suggested that they divide at very similar rates. The average telomere length of naive $\mathrm{CD} 4{ }^{+} \mathrm{T}$ lymphocytes was consistently found to be $1.4 \mathrm{~kb}$ longer than that of their memory counterparts (Fig. 1). It was therefore concluded that the magnitude of expansion occurring when naive $\mathrm{CD} 4^{+} \mathrm{T}$ cells are primed to become memory cells is $1400 / 100=14$ divisions. Additionally, the fact that naive telomeres shorten with age was taken as evidence that naive $\mathrm{T}$ cells divide throughout their lifespan: a loss of $1.5 \mathrm{~kb}$ in 45 years was estimated to amount to $1500 /(100 \times 45)=0.3$ cell divisions/year. This estimate is in good agreement with previous studies on naive lymphocyte turnover, which were based on the rate at which patients treated

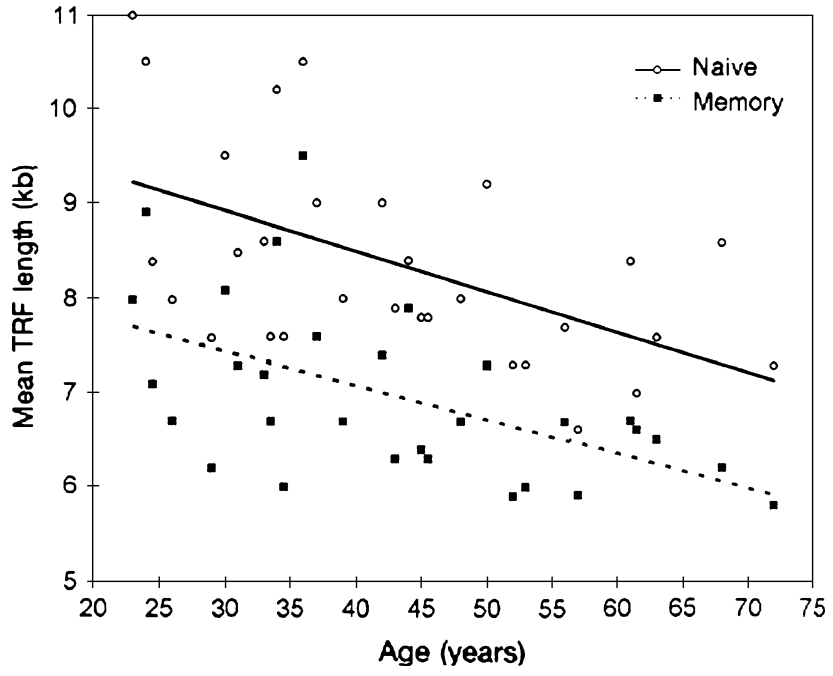

Fig. 1. Comparison of the mean TRF length of naive and memory $T$ cells. Thirty donors were studied, ranging in age from 24 to 72 years. Open circles denote the mean TRF length of each individual's naive T cells; the solid line represents the regression analysis of the TRF lengths of naive cells as a function of age; solid squares denote the mean TRF length of each individual's memory T cells; the dotted line represents the regression analysis of the TRF lengths of memory cells as a function of age. Reprinted with permission from Weng et al.(22). Copyright 1995, The National Academy of Sciences. TRF, telomere restriction fragment.

with radiotherapy lose lymphocytes with chromosomal damage (23). The latter studies showed that CD45RA naive T cells divide once every 3.5 years, whereas memory lymphocytes divide once every 22 weeks $(23,24)$. Remarkably, the fact that naive $\mathrm{CD} 4{ }^{+} \mathrm{T}$ lymphocytes are thought to proliferate much less than memory $\mathrm{CD}^{+}{ }^{+} \mathrm{T}$ lymphocytes did not at all seem to be reflected in the telomere data, which intuitively suggested similar kinetics of naive and memory lymphocytes (22).

A mathematical model for telomere loss (Box 1) helped understand the apparent controversy (25). The model pointed out that the intuitive derivation of the rate of naive $\mathrm{T}$-cell turnover from the telomere data (22) was wrong for several reasons. Among others, it was shown that the average telomere length decreases at twice the average division rate because every parent cell is replaced by two daughter cells with shortened telomeres. Thus, with a telomere loss of 100 base pairs/cell division, 0.15 divisions/year would suffice to observe a loss of the average naive telomere length of $1.5 \mathrm{~kb}$ in 45 years (25). Additionally, Weng et al. (22) ignored the export of newly produced $\mathrm{T}$ cells from the thymus into the periphery. Because recent thymus emigrants form a permanent source of cells with relatively long telomeres, they increase the average telomere length of the naive T-cell population. Ignoring thymic output may therefore lead to an underestimation of the naive T-cell division rate. 
Box 1. A mathematical model for telomere loss in $\mathrm{T}$ cells

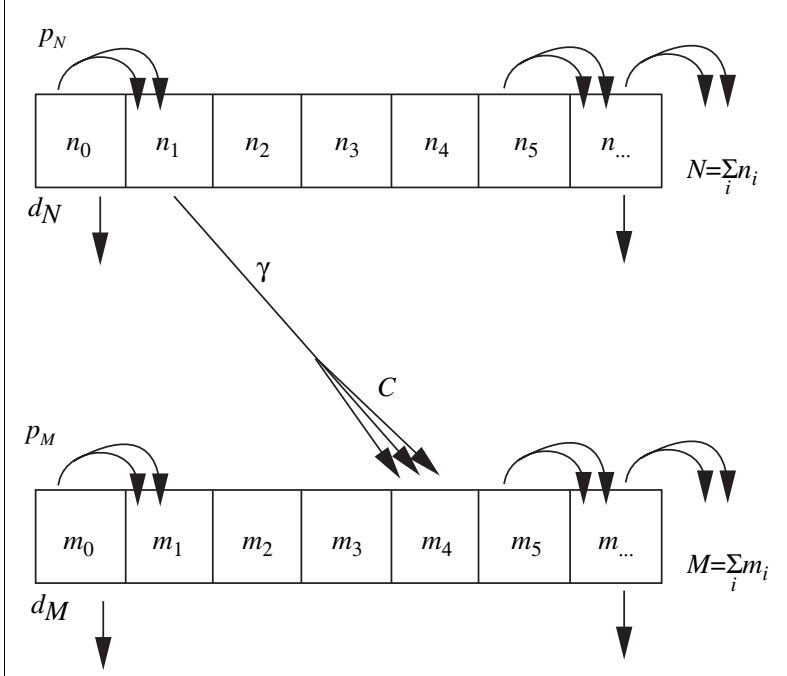

Fig. Box 1. A scheme of the model. The boxes $n_{i}$ and $m_{i}$ represent the number of naive and memory $\mathrm{T}$ cells that have gone through precisely i cell divisions, respectively. $\mathrm{N}$ and $\mathrm{M}$ are the total number of naive and memory $\mathrm{T}$ cells, that is $\mathrm{N}=\sum_{\mathrm{i}} \mathrm{n}_{\mathrm{i}}$ and $\mathrm{M}=\sum_{\mathrm{i}} \mathrm{m}_{\mathrm{i}}$, respectively. The arrows $\mathrm{p}$ and $\mathrm{d}$ represent division and death rates, respectively. The arrow $\gamma C$ represents the influx of naive cells into the memory pool through priming and proliferation during immune responses to foreign antigens. Adapted from De Boer and Noest (25).

In the model (25), the differential equation for the average telomere loss index $(\mu)$ of naive T cells $(\mathrm{N})$ and memory $\mathrm{T}$ cells $(\mathrm{M})$ is:

$$
\frac{\mathrm{d} \mu_{N}}{\mathrm{~d} t}=2 p_{N} \text { and } \frac{\mathrm{d} \mu_{M}}{\mathrm{~d} t}=2 p_{M}-\gamma C \frac{N}{M}\left(\mu_{M}-\mu_{N}-K\right),
$$

where $\mathrm{K}$ is the telomere loss during clonal expansion, $\gamma$ is the rate at which naive $\mathrm{T}$ cells are primed into the memory pool, and $\mathrm{C}$ is the average number of cell divisions occurring during clonal expansion (25). Eqn 1 shows that the telomere loss caused by memory T-cell proliferation is diminished by activation of naive cells that are primed into the memory pool and, on average, have longer telomeres than memory cells. The difference between the average telomere loss index of naive and memory cells $\left(\Delta=\mu_{\mathrm{M}}-\mu_{\mathrm{N}}\right)$ will ultimately approach the steady state:

$$
\Delta=K+\frac{2\left(p_{M}-p_{N}\right) M}{\gamma C N},
$$

that is the difference between the average telomere index of naive and memory cells is only partially determined by the telomere loss during clonal expansion. It also reflects the difference in proliferation rates of naive and memory cells ( $p_{N}$ and $p_{M}$ ) and their densities ( $N$ and $M$ ). Even if telomerase compensates completely for the telomere loss during clonal expansion $(K=0)$, there is an age-independent difference between the average telomere length of naive and memory $\mathrm{T}$ cells, provided that memory cells divide more frequently than naive cells $\left(p_{M}>p_{N}\right)$. Once the equilibrium of Eqn 2 is approached, filling in Eqn 2 into Eqn 1 shows that the rate of telomere loss in the memory population is completely dictated by the rate of telomere loss in the naive T-cell pool, that is $\mu_{\mathrm{M}}=\mu_{\mathrm{N}}+\Delta$.

The above example also shows the difficulties in estimating the proliferation rate of memory $\mathrm{T}$ cells from telomere data. Just like the average telomere loss of naive $\mathrm{T}$ cells is diminished by the influx of newly produced cells from the thymus, the average telomere loss of memory cells is diminished by activation of naive cells that are primed into the memory pool and, on average, have longer telomeres than memory $\mathrm{T}$ cells. Indeed, the mathematical model showed that the telomere shortening rate of memory cells does not only reflect their own division rate but also critically depends on the rate at which naive cells are primed into the memory pool, the loss of telomere length during clonal expansion, the ratio between naive and memory lymphocyte numbers, and the difference in the average telomere length of naive and memory lymphocytes (25) (Box 1, Eqn 1).

The mathematical model also pointed out that the difference between the average telomere length of naive and memory cells does not directly reflect the clonal expansion that naive $\mathrm{CD} 4{ }^{+} \mathrm{T}$ cells undergo when they are primed to become memory cells but also reflects the difference in proliferation rates of naive and memory $\mathrm{T}$ cells (Box 1, Eqn 2). If memory $\mathrm{T}$ cells divide more frequently than naive $\mathrm{T}$ cells, the 14 divisions that were estimated to occur during clonal expansion (22) only provide an upper-bound estimate. Interestingly, the model shows that even if no telomere shortening were to occur during clonal expansion, the average telomere length of naive and memory $\mathrm{T}$ cells would approach a fixed difference (25). It has indeed been suggested that hardly any shortening of telomeres may occur during clonal expansion because telomerase, an enzyme that is able to extend shortened telomere ends, has been shown to be active in lymphocytes $(26,27)$.

According to the mathematical model, the fact that there is an age-independent difference in naive and memory telomere 
lengths (22) implies that the average rate at which memory lymphocytes lose their telomeres is independent of their own division rate and is largely dictated by the rate of telomere loss in the naive T-cell compartment (Box 1). This insight resolves the apparent contradiction between the parallel loss of telomeres in naive and memory $\mathrm{T}$ lymphocytes and the fact that memory $\mathrm{T}$ cells are thought to divide more frequently than naive $\mathrm{T}$ cells.

\section{Estimating thymus output from TREC data}

The above-mentioned problems in the interpretation of telomere data stress the need to quantify all different processes that contribute to peripheral T-cell kinetics. One process that has been difficult to quantify is the output of newly produced $\mathrm{T}$ cells from the thymus. Previous studies established that naive T cells are largely generated in the thymus, but after thymectomy in mice, naive $\mathrm{T}$ cells can be maintained by peripheral proliferation (28-31). Recent studies in humans have suggested that naive Tcell proliferation may contribute significantly to the generation and maintenance of the naive T-cell pool (32). A large step forward was made by the introduction of the TREC assay (33, 34). TRECs are extrachromosomal DNA circles, which are formed as a by-product during T-cell receptor gene rearrangement in the thymus and are not copied during $\mathrm{T}$-cell division. When the average number of TRECs per $\mathrm{CD} 4^{+}$or $\mathrm{CD} 8^{+} \mathrm{T}$ cell was measured in individuals of different ages, an exponential loss of TRECs was observed, reminiscent of the supposedly exponential decay of thymus output with age (34). TREC contents of $\mathrm{T}$ lymphocytes have therefore widely been used to measure thymus output. For example, the fact that TREC contents of $\mathrm{CD}^{+}{ }^{+}$and $\mathrm{CD} 8{ }^{+} \mathrm{T}$ cells tend to decline during HIV infection has been taken as evidence for HIV-induced loss of thymus output (34), and the increase in $\mathrm{CD}^{+}$and $\mathrm{CD} 8^{+} \mathrm{TREC}$ contents that are generally observed following stem cell transplantation have been interpreted as evidence for thymus rebound (35).

The advantage of TRECs is that their production is confined to $\mathrm{T}$-cell receptor gene rearrangements and thereby to de novo naive T-cell generation. In contrast to naive T cells themselves, TRECs cannot be formed through cellular division. Unfortunately, TRECs are usually measured as a fraction of the amount of DNA analyzed and are therefore typically expressed as TREC content (i.e. TREC numbers per cell), a measure that is to a large extent influenced by T-cell division. Another difficulty in the interpretation of TREC data is that TRECs are known to be longlived and are thus not a good marker of current thymus output. Indeed, in patients who had been fully thymectomized, TRECs could still be identified in $\mathrm{CD}^{+}$and $\mathrm{CD} 8^{+} \mathrm{T}$ cells up to 39 years after thymectomy (34). Intuitive interpretation of TREC data may therefore easily lead to false conclusions $(15,36)$.

A simple mathematical model describing the kinetics of naive $\mathrm{T}$ cells and TRECs showed that an age-dependent decrease in thymus output is not sufficient to explain the decrease in naive $\mathrm{CD}^{+}$and $\mathrm{CD} 8^{+}$TREC contents with age $(37,38)($ Box 2$)$. In fact, the TREC content of a T-cell population that is dependent on the influx of cells from the thymus may be totally insensitive to changes in thymus output (Fig. 2). With hindsight, this
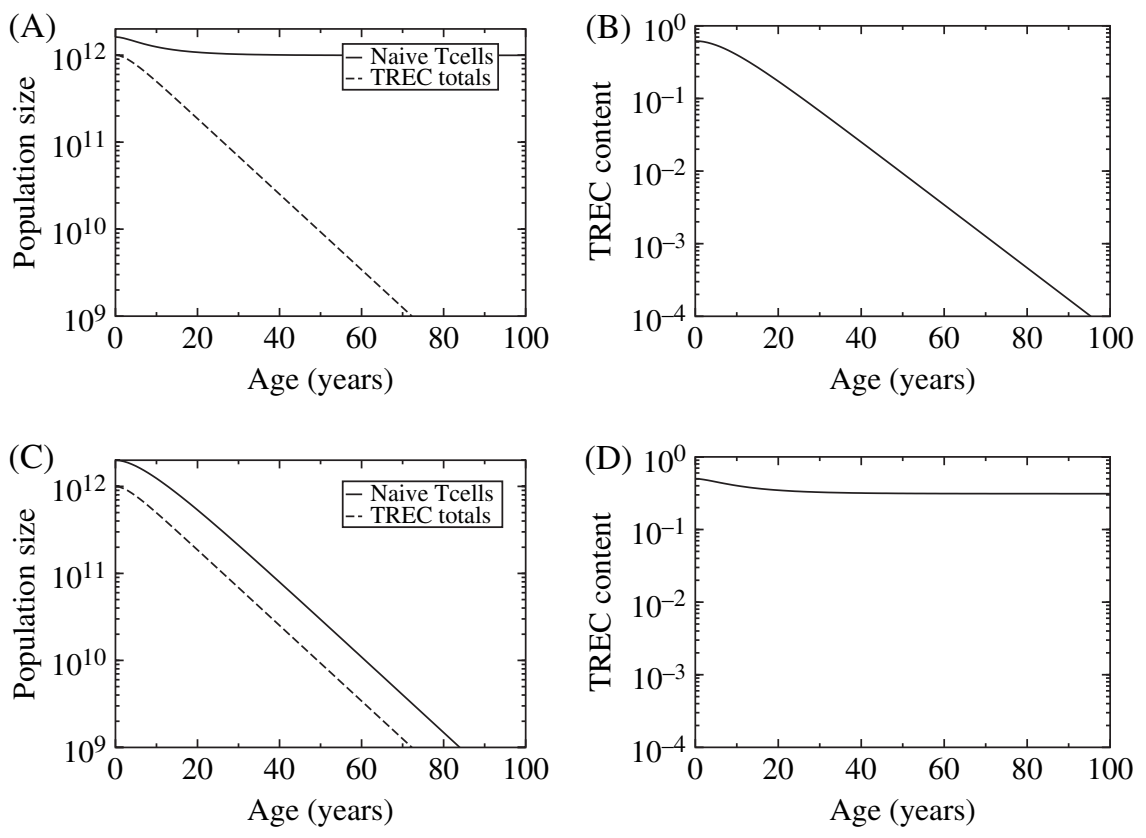

Fig. 2. Naive T-cell numbers (N), total TREC numbers (T), and TREC contents $(A)$ as a function of age, when thymus output decreases exponentially with age. (A, B) In the presence of homeostatic proliferation or death, the TREC content declines significantly with age (B) because naive T-cell numbers are less affected by the involution of the thymus than total TREC numbers (A). (C, D) In the absence of homeostatic processes within the naive T-cell pool, the naive TREC content only changes somewhat with age during an initial transient. After this transient, the total number of TRECs and naive $T$ cells decrease at the same rate (C) and the TREC content (D) approaches a fixed fraction. Model and parameters as in Hazenberg et al. (37): $\sigma(\mathrm{t})=$ $10^{9} \mathrm{e}^{-0.1 a}$ cells/day, where a is age in years, $c=1, d=0.001 /$ day, and $p=0.1 /(1+$ $\left.\left(\mathrm{N} / 2.5 \times 10^{10}\right)^{2}\right)$ per day $(\mathrm{A}$ and $\mathrm{B})$ or $\mathrm{p}=$ $0.0005 /$ day (C and D) (see also Box 2). 


\section{Box 2. Mathematical model for TREC analysis}

In the model for TREC analysis by Hazenberg et al. (37), the dynamics of naive $\mathrm{T}$ cells was described as:

$$
\frac{\mathrm{d} N}{\mathrm{~d} t}=\sigma(t)+p N-d N
$$

where $\sigma(\mathrm{t})$ is an age-dependent thymic production, $\mathrm{p}$ is the renewal rate, and $d$ is the death rate of naive $T$ cells. The dynamics of the total number of TRECs was described as:

$$
\frac{\mathrm{d} T}{\mathrm{~d} t}=c \sigma(t)-d T
$$

where $c$ is the TREC content of a recent thymic emigrant. From these equations, one can derive that the TREC content $(A=\mathrm{T} / \mathrm{N})$ changes according to:

$$
\frac{\mathrm{d} A}{\mathrm{~d} t}=\frac{\sigma(t)(c-A)}{N}-p A,
$$

so that the equilibrium of the TREC content is given by:

$$
A=\frac{c}{1+p N / \sigma(t)}
$$

counterintuitive finding is easy to understand. In a population that is dependent on thymus output, not only the number of TRECs but also the number of cells decreases as thymus output declines, and their ratio could remain unaffected. The fact that naive $\mathrm{CD}^{+}{ }^{+}$and $\mathrm{CD} 8{ }^{+}$TREC contents do decrease with age (39) thereby shows another aspect of T-cell dynamics: according to the mathematical model, an age-dependent naive TREC decline can only occur if there is at least some form of homeostasis in the naive T-cell pool (38). If the body responds to the agedependent decline in thymus output, for example by increasing the level of naive T-cell proliferation, the total number of TRECs will decline faster than the total number of naive $T$ cells, causing an age-dependent decline in the average TREC content of naive T cells. Alternatively, the level of naive T-cell proliferation may be constant, and cells may simply survive longer when thymus output declines, which would also lead to a decline in naive TREC contents with age (38).

The above example shows how strongly TREC content data may be influenced by cellular division rates and the care that should hence be taken when interpreting TREC content data. The decrease in TREC content that is typically observed on HIV infection, for example, may not at all reflect a decrease in thymus output but instead may result from the increased level of T-cell activation that is characteristic for HIV infection (37). Similarly, increases in TREC contents that have been observed in patients with lymphopenia and have been interpreted as evidence for thymic rebound, that is an increased rate of thymus export to compensate for low T-cell numbers (34), may in fact reflect the normal influx of recent thymus emigrants into a virtually empty peripheral T-cell pool that has not yet diluted its TREC content (37). Additionally, the fact that TREC contents are often measured in peripheral blood mononuclear cells or in total $\mathrm{CD}^{+}$or $\mathrm{CD}^{+} \mathrm{T}$ cells hampers the interpretation because changes in the ratio of naive and memory cells may strongly influence the average TREC content within these populations (15).

In contrast to TREC contents, total TREC numbers per milliliter of blood are not influenced by T-cell proliferation and hence provide a more direct measure of thymus output $(15,40$, 41). When analyzing thymus output, it would therefore be good common practice to analyze total TREC numbers in addition to TREC contents $(15,40)$. However, even TREC totals have to be taken with care because they are affected not only by thymus output but also by changes in T-cell death rates (Box 2). Remarkably, in the literature, the use of telomeres is typically restricted to measuring T-cell division, whereas TREC data are quite strictly used to measure thymus output. The above models $(25,37,38)$ show, however, that in fact, both telomere and TREC data are influenced by thymus output and T-cell proliferation.

\section{BrdU labeling to measure lymphocyte turnover}

T-cell turnover rates have extensively been studied by the use of BrdU, a nucleoside analogue that is incorporated instead of thymidine through the nucleotide salvage pathway when a cell divides. BrdU has widely been applied in rodents and nonhuman primates, but its potential toxicity on long-term in vivo administration has limited its use in humans to short-term labeling periods (42-45). The use of $\mathrm{BrdU}$ is relatively straightforward, as it can be administered through the drinking water and as its incorporation in cells can be detected by flowcytometry. Obtaining T-cell turnover rates from BrdU-labeling data, however, has turned out to be less straightforward, and BrdU-labeling data have long been interpreted rather qualitatively $(46,47)$. The fraction of cells that have acquired BrdU during label administration and the rate at which labeled cells lose BrdU after label cessation are often intuitively taken to reflect the fractions of cells that have undergone proliferation 
and death, respectively. For example, Kovacs et al. (43) observed a bi-phasic loss of BrdU-labeled cells during the down-labeling phase, and they fitted two exponential slopes to the data. These slopes were interpreted as the death rates of two distinct subpopulations.

Mathematical models have pointed out that in fact, the labeling and the delabeling curve are influenced by both T-cell proliferation and cell loss $(1,48)$. Indeed, from the model described in Box 3, one can observe that the rate at which the fraction of labeled cells increases during label administration is equal to the sum of the proliferation rate and the rate at which cells are lost from the population through cell death or maturation. Naturally, the rate at which the fraction of labeled cells increases is equal to the rate at which the fraction of unlabeled cells decreases, and unlabeled cells are lost from the population not only on cell division but also on cell death or maturation. In analogy, the rate at which the fraction of labeled cells decreases after label cessation gives the difference between the rate of cell loss and proliferation, not directly the rate of cell loss (Box 3). The latter is because of the fact that BrdU-labeled cells that proliferate in the absence of label still give rise to two labeled daughter cells. This leads to the paradoxical insight that a T-cell population in which the fraction of labeled cells hardly decreases after label cessation may be a population of long-lived cells but could just as well be a cell population with rapid turnover, in which high rates of T-cell proliferation are compensated by high rates of T-cell death or maturation. Only after extensive T-cell proliferation in the absence of label, BrdU labeling may have diluted to such an extent that cells are no longer picked up as label positive. In this case, the fraction of labeled cells would be underestimated, which causes another difficulty in the interpretation of BrdU-labeling data, for which more extensive mathematical models have been developed (48).

Given the fact that the rate at which BrdU labeling is lost after label cessation is dictated by the difference between lymphocyte proliferation and loss ( $p-d$, Box 3$)$, one would expect to see hardly any loss of BrdU-labeled cells after stopping label administration because in a $\mathrm{T}$-cell population at steady state, the rate at which cells proliferate (p) and the rate at which cells are lost from the population (d) should be nearly identical. It is therefore rather surprising that most BrdU studies show a significant decline during delabeling, suggesting that the average proliferation rate of lymphocytes ( $p$ ) should be smaller than the average loss rate of BrdU-labeled cells (d). Indeed, separate estimates of $d$ and $p$ have shown up to 10 -fold larger rates of cell loss compared with the rate of $\mathrm{T}$-cell proliferation (1). It has been proposed that this discrepancy between cell loss

\section{Box 3. Mathematical model for BrdU labeling}

A mathematical model for BrdU labeling (1, 11, 12, 48) describes the dynamics of unlabeled cells, $U$, and labeled cells, L, during the up-labeling phase by

$$
\frac{\mathrm{d} U}{\mathrm{~d} t}=-p U-d U \text { and } \frac{\mathrm{d} L}{\mathrm{~d} t}=s+2 p U+p L-d L .
$$

In the presence of BrdU, proliferation (at rate p per day) of an unlabeled cell leads to the loss of that unlabeled cell and the formation of two labeled cells because BrdU is built into one strand of DNA of each chromosome. The net result of proliferation of a labeled cell, on the other hand, is the formation of just one extra labeled cell. Both unlabeled and labeled cells are lost at rate d per day through T-cell death or maturation to another $\mathrm{T}$-cell population. In addition, labeled cells may be generated from a source at rate s cells per day.

During the down-labeling phase, the dynamics are given by:

$$
\frac{\mathrm{d} U}{\mathrm{~d} t}=s+p U-d U \text { and } \frac{\mathrm{d} L}{\mathrm{~d} t}=p L-d L .
$$

In the absence of BrdU, proliferation of an unlabeled cell adds another unlabeled cell to the population, whereas proliferation of a labeled cell adds a labeled cell (albeit with lower BrdU intensity) because the previously labeled chromosomes are randomly distributed over both daughter cells. Both unlabeled and labeled cells are lost from the population at rate $d$. Unlabeled cells may additionally be generated from a source at rate s cells per day.

To describe the fraction of labeled cells $(L / L+U))$, one can use $\mathrm{dU} / \mathrm{dt}=-(\mathrm{p}+\mathrm{d}) \mathrm{U}$ during the up-labeling phase and $\mathrm{dL} / \mathrm{dt}=(p-\mathrm{d}) \mathrm{L}$ during the down-labeling phase. This shows that the upslope is determined by $p+d$, whereas the downslope represents $p-d$. Note that if unlabeled and labeled cells are lost from the population at different rates, for example because recently divided cells die faster than cells that have not recently divided (57), the rate of label accrual during BrdU administration should be calculated from the more complicated equation for $\mathrm{dL} / \mathrm{dt}$ (Eqn $7 \mathrm{~b}$ ), as was performed by Debacq et al. (49). If one adds a parameter $C$ for the maximum labeling level (1), the initial upslope is $C(p+d)$ per day.

and proliferation may be compensated for by a constant source of T cells, for example from the thymus or from a compartment of resting cells, and special models have been designed to account for such a source $(1,11,12,48)$. 
When the model described in Box 3 was used to calculate proliferation and death rates of $\mathrm{T}$ cells in healthy and simian immunodeficiency virus-infected rhesus macaques, large confidence intervals on the estimated rates of T-cell proliferation were obtained (1). Such large confidence intervals may well be as a result of the sensitivity of the parameter estimates on the underlying assumptions of the specific mathematical model used (11). For instance, having both a source and a proliferation term for the production of new cells allowed for compensation between these parameters, which implies that the original estimates for s and p (1) were unreliable $(11,12)$. Extensive comparisons of the parameter estimates obtained from very different mathematical models for BrdU labeling have pointed out, however, that one parameter, the socalled average turnover rate (i.e. the cellular death rate averaged over all subpopulations), can be determined with much better reliability and thereby provides the most robust and modelindependent parameter from BrdU-labeling data $(11,12)$. Summarizing, the pioneering work on developing the most appropriate mathematical models for estimating average cellular turnover rates from long-term BrdU data $(1,48)$ seems to have converged on simple models with just one or two parameters $(11,49)$, and these simple models have allowed us to reliably estimate the average turnover rates of naive and memory $\mathrm{CD}^{+}$and $\mathrm{CD} 8^{+} \mathrm{T}$ cells, natural killer cells, and B cells (12).

\section{Labeling with stable isotopes to measure lymphocyte turnover}

An important shortcoming of BrdU is its potential toxicity on long-term administration and hence the limitations of its use in human subjects. More recently, new methods have been developed by which T-cell turnover can be measured using stable-isotope-labeled compounds, such as deuterated glucose $\left({ }^{2} \mathrm{H}_{2}\right.$-glucose) (50) or deuterated water $\left({ }^{2} \mathrm{H}_{2} \mathrm{O}\right)$ (51), which are incorporated into the DNA of newly dividing cells. After label intake, cells are sorted from peripheral blood; their genomic DNA is extracted, hydrolyzed, and derivatized; and the fraction of ${ }^{2} \mathrm{H}$ incorporation in the deoxyribose moiety of deoxyadenosine is subsequently analyzed by a combination of gas chromatography and mass spectrometry (52). The advantages of the method are several. First, in contrast to BrdU labeling, ${ }^{2} \mathrm{H}$ labeling measures de novo nucleotide synthesis, thereby capturing the major component of lymphocyte proliferation, and is relatively insensitive to label reutilization. Second, the use of stable isotopes circumvents the radiationinduced DNA damage and potential toxic effects that are intrinsic to the use of radioisotopes and nucleoside analogues (52). Third, stable isotope labeling simplifies the interpretation of the data because the fraction of labeled DNA strands rather than the fraction of labeled cells is measured. The decay of labeled DNA after cessation of stable isotope administration thereby directly reflects the loss of cells (see below). In contrast to BrdU labeling, cell division after label cessation can no longer lead to label enrichment.

Stable-isotope-labeling data have been interpreted in various ways. The most widely used method is the application of the precursor-product relationship that has been adopted from classical protein chemistry $(50,52)$ :

$$
f=\frac{S_{\mathrm{b}}}{S_{\mathrm{a}}}=1-\mathrm{e}^{-k t} .
$$

The equation describes that the fraction $f$ of new cells generated within a time period $\mathrm{t}$ can be derived from the label enrichment $S_{\mathrm{b}}$ measured in the DNA of the cell population of interest (the product), divided by the label enrichment $S_{\mathrm{a}}$ of the precursor. In case of labeling with deuterated glucose, the precursor enrichment is typically measured as the label enrichment in plasma glucose multiplied by a factor 0.65 to account for intracellular label dilution (52). In case of labeling with deuterated water, $S_{\mathrm{a}}$ is derived from the label enrichment of cells with extremely rapid turnover, such as monocytes or granulocytes (51). The precursor-product relationship shows the fractional replacement rate $k$ and thereby the half-life (ln2/ k) of the cells of interest. A limitation of the application of the precursor-product relationship in biology is the fact that replacement is assumed to occur randomly, as in chemical processes. The kinetics of the cell population of interest, however, may not be as homogeneous as a pool of identical molecules.

Hellerstein et al. (2) used the precursor-product relationship to interpret data from 2-day labeling with deuterated glucose, and they estimated that $\mathrm{CD}^{+}$and $\mathrm{CD} 8^{+} \mathrm{T}$ cells in healthy subjects have half-lives of 87 and 77 days, respectively. Using the same method, McCune et al. (53) estimated a longer half-life of $\mathrm{CD}^{+}{ }^{+} \mathrm{T}$ cells: $\mathrm{CD} 4^{+}$and $\mathrm{CD} 8^{+} \mathrm{T}$ cells were estimated to have half-lives of 82 and 139 days, respectively. Separate analyses of naive and effector/memory (E/M) cells showed that E/M T cells have a considerably shorter half-life than naive T cells (53) (Table 1). A drawback of both studies is that there was no clear distinction between the phases of accumulation and loss of isotope enrichment. The peak in label enrichment could occur up to 10 days after cessation of label infusion. To estimate fractional replacement rates, both studies therefore used the maximum level of enrichment that was ever attained after label 
Table 1. Average half-lives (in days) of different T-cell populations in healthy individuals estimated by stable isotope labeling

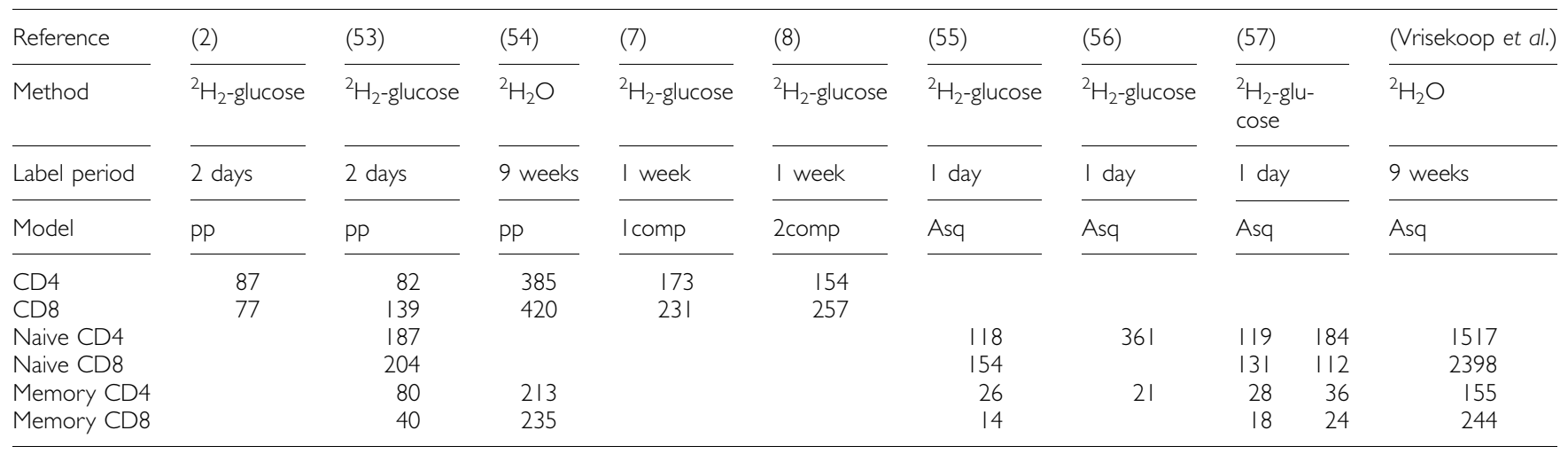

The half-lives have been recalculated from previous studies $(2,7,8,53,54,55-57)$. For every model distinguishing between proliferation and death rates, we based the half-life on the proliferation rate (which is mostly based on the upslope during the labeling period) because the downslope reflects the turnover rate of labeled cells $(6,58)$, which tends to be faster than the average turnover rate. pp, precursor-product relationship; I comp and 2 comp, Iand 2-compartment mathematical models, respectively; Asq, the model by Asquith et al. (6) accounting for cellular heterogeneity. The two columns under ref (57) refer to T-cell half-lives measured in young and elderly individuals, respectively.

infusion, thereby losing all information from other data points, making the estimates vulnerable to variation.

More recently, Hellerstein et al. (54) presented the results of the first long-term labeling experiment based on 9 weeks of administration of deuterated water. The non-linear accrual of isotope label that was observed during administration of ${ }^{2} \mathrm{H}_{2} \mathrm{O}$ was intuitively interpreted to reflect the turnover of different subpopulations within the total and $\mathrm{E} / \mathrm{M} \mathrm{CD}^{+}$and $\mathrm{CD}^{+}{ }^{+}$T-cell pools. However, even based on the precursorproduct relationship, a non-linear accrual of label would be expected. Indeed, using Eqn 9 to estimate the cellular half-lives from the level of enrichment attained after 5 and 9 weeks of label administration gives very similar estimates for the replacement rate $\mathrm{k}$. The estimated half-lives of total $\mathrm{CD} 4^{+}$ (385 days), total $\mathrm{CD} 8^{+}$(420 days), E/M CD $4^{+}$(213 days), and $\mathrm{E} / \mathrm{M} \mathrm{CD}^{+}$(235 days) $\mathrm{T}$ cells based on this study were, however, several folds larger than those of the previous studies based on deuterated glucose labeling (Table 1).

An important limitation of the use of the precursor-product relationship in the interpretation of labeling data is that it only measures the net accrual of label. Cells that have taken up label during division but are lost from the population during the labeling period do not contribute to the turnover measured by the precursor-product relationship. The use of the precursorproduct relationship is therefore expected to underestimate the extent of T-cell turnover, especially during long-term labeling, when the chance to loose labeled cells during the labeling period is large. Although the single parameter $\mathrm{k}$ that can be estimated by the use of the precursor-product relationship is a natural parameter in terms of protein chemistry, it may be very hard to interpret in immunological terms. A perfect illustration of this problem was given when stable-isotope-labeling data from healthy individuals and HIV-infected patients with and without therapy were compared (2). The maximal level of label enrichment that was achieved after 2-day labeling with deuterated glucose was found to be the lowest in healthy individuals and the highest in patients with HIV on highly active anti-retroviral treatment (HAART). As a result, the replacement rate $\mathrm{k}$ was found to be higher in patients on HAART than in untreated patients with HIV and healthy individuals. From these data, the paradoxical conclusion was drawn that the half-life of $\mathrm{T}$ cells in patients on HAART is shorter than in untreated patients and that the mechanism by which HAART increases the $\mathrm{CD} 4{ }^{+} \mathrm{T}-$ cell count in patients with HIV is not through increased $\mathrm{CD} 4^{+} \mathrm{T}$ cell survival but by increased production of new $\mathrm{CD} 4^{+} \mathrm{T}$ cells (2). Moreover, these data were taken as evidence for increased $\mathrm{CD} 4{ }^{+}$T-cell production during HAART because the absolute $\mathrm{CD} 4{ }^{+} \mathrm{T}$-cell production rate, that is $\mathrm{k}$ multiplied by the $\mathrm{CD} 4{ }^{+} \mathrm{T}$ cell count, was found to be higher in patients on HAART than in untreated patients. Analogously, it was argued that HIV infection decreases the half-life of $\mathrm{CD}^{+}{ }^{+} \mathrm{T}$ cells and that there is no increased $\mathrm{CD}^{+}{ }^{+} \mathrm{T}$-cell production to compensate for this reduced survival because $\mathrm{k}$ was higher in patients with HIV than in healthy individuals, whereas the absolute $\mathrm{CD}^{+} \mathrm{T}$-cell production rate was not. These interpretations may fail for two reasons. First, the absolute $\mathrm{CD} 4^{+} \mathrm{T}$-cell production rates that were calculated are largely determined by the $\mathrm{CD} 4^{+} \mathrm{T}$-cell counts of the individuals, and it may thus not be surprising that they were higher in patients on HAART than in untreated patients. Second, high levels of enrichment may be as a result of high levels of T-cell proliferation or increased survival of cells that have recently picked up label (see below). The fact that 
patients with HIV reached higher levels of label enrichment than healthy individuals may therefore be because of increased rates of $\mathrm{T}$-cell proliferation, which have indeed frequently been observed in patients with $\operatorname{HIV}(7,37,59,60)$, whereas the even higher levels of enrichment in patients on HAART may be because of increased survival of recently divided cells (8). Both conclusions conflict with those originally drawn from the data (2).

\section{Mathematical models distinguishing between proliferation and cell loss}

Mathematical models have helped interpret stable isotope data. By fitting labeling data both during and after label administration to a mathematical model, separate estimates of the proliferation and loss rates of the different T-cell populations have been obtained. Based on such a mathematical model, label enrichment in $\mathrm{CD}^{+}$and $\mathrm{CD}^{+} \mathrm{T}$ cells in healthy humans was analyzed during and after 7-day infusion of ${ }^{2} \mathrm{H}_{2}$-glucose (7). $\mathrm{CD}^{+}$and $\mathrm{CD} 8^{+} \mathrm{T}$ cells were found to have average proliferation rates of 0.004 and 0.003 /day, whereas their loss rates were as high as 0.044 and 0.043 /day, respectively. Because T-cell numbers were not changing over the course of the experiment, the rates of $\mathrm{T}$-cell proliferation and loss a priori were expected to be nearly identical. The discrepancies between relatively low proliferation rates and high loss rates were explained by the presence of a large source of unlabeled cells (7). It was this source of unlabeled cells, however, that was highly criticized in the literature: the estimated contribution of the source of unlabeled cells would have to be about 5-30 times higher than the contribution of T-cell proliferation. If the source of unlabeled cells were to come from the thymus, it would require a thymus output of $10^{10}$ instead of the previously estimated $10^{8} \mathrm{~T}$ cells/day. Alternatively, it has been proposed that a large source of unlabeled cells may come from a compartment of resting cells (7-9). Fitting a model including such a population of resting cells to the same data led to very similar estimates for the proliferation and loss rates of $\mathrm{CD} 4^{+}$and $\mathrm{CD}^{+}{ }^{+} \mathrm{T}$ cells $(8,9)$ (Table 1$)$.

With hindsight, the common observation with both deuterium and BrdU labeling that the estimated loss rate of lymphocytes exceeds their proliferation rate is not all that surprising. Proliferation rates, which are estimated during label administration, provide a characteristic of the population as a whole, including cells that will and cells that will not go into division during the labeling period. Loss rates, in contrast, are based on the loss of cells that have picked up the label and hence only involve the part of the lymphocyte pool that has recently divided $(6,8,58)$. Ribeiro et al. (8) captured part of this heterogeneity in their model by distinguishing between resting and activated cells. Proliferation and loss rates within the activated pool were assumed to be equal to allow for a constant population size, but the proliferation rate of the total T-cell pool was again several folds lower than the loss rate of the labeled cells.

In an illuminating article on the interpretation of labeling data, Asquith et al. (6) proposed a more rigorous approach to account for this heterogeneity. It was argued that even modeling two compartments may be insufficient to fully capture the effects of cellular heterogeneity because the kinetics of cells that have picked up label may be intrinsically different from those that have failed. First, the labeled fraction is expected to be biased toward the part of the T-cell population with the most rapid kinetics. Second, cells that have recently divided are likely to be lost from the population at a higher rate than those that have not picked up the label because they are more likely to undergo activation-induced cell death (58). To cope with this cellular heterogeneity, a mathematical model was introduced in which the proliferation rate $p$ of the whole T-cell population was uncoupled from the loss rate of labeled cells $d^{*}$ :

$$
f=\frac{p}{d^{*}}\left(1-\mathrm{e}^{-d^{*} t}\right) \text { and } f=\frac{p}{d^{*}}\left(1-\mathrm{e}^{-d^{*} \tau}\right) \mathrm{e}^{-d^{*}(t-\tau)},
$$

during and after labeling, respectively (6). The model describes the accrual of label during label administration as well as the loss of enrichment after label cessation at day $\tau$. The first equation is very similar to the widely used precursor-product relationship, but it now distinguishes between proliferation and cell loss and saturates at a lower fraction of labeled cells, that is when a fraction $\mathrm{p} / \mathrm{d}^{*}$ of the cells is labeled. Both equations become identical if $p=d^{*}$, in other words, if the loss rate of labeled cells is identical to that of the non-labeled cells and hence if the T-cell population is fully homogeneous. Stable-isotope-labeling data have shown, however, that this is typically not the case, underscoring the limitations of the use of the precursorproduct relationship.

The above kinetic heterogeneity model has given the additional insight that the parameter estimates resulting from labeling data depend on the length of the labeling period (6). Long labeling periods will give rise to lower rates of T-cell loss because the population that has picked up the label becomes more representative of the total T-cell population. Indeed, metaanalysis of stable-isotope-labeling studies with different labeling periods showed such a negative correlation between the length of the labeling period and the estimated death rate (6). 
Based on the above kinetic heterogeneity model, T-cell turnover in healthy individuals was estimated following 1 day of ${ }^{2} \mathrm{H}_{2}$-glucose labeling, showing proliferation rates of naive $\mathrm{CD}^{+}\left(0.6 \%\right.$ per day), naive $\mathrm{CD}^{+}(0.5 \%$ per day $)$, memory $\mathrm{CD}^{+}\left(2.7 \%\right.$ per day), and memory $\mathrm{CD}^{+}(5.1 \%$ per day) $\mathrm{T}$ cells (55) that were higher than previous estimates based on the precursor-product relationship (53). In two later studies, the same mathematical model (6) and a similar 1-day infusion of ${ }^{2} \mathrm{H}_{2}$-glucose were used to study the turnover rates of naive $\mathrm{CD}^{+}{ }^{+} \mathrm{T}$ cells, $\mathrm{CD} 4{ }^{+} \mathrm{CCR} 7^{+}$effector-memory (EM) $\mathrm{T}$ cells, and $\mathrm{CD} 4{ }^{+} \mathrm{CCR} 7{ }^{-}$central memory (CM) T cells (56) and of naive and memory $\mathrm{T}$ cells in young and elderly people (57). Turnover rates of naive $\mathrm{CD}^{+}{ }^{+}\left(0.2 \%\right.$ per day), $\mathrm{CD} 4{ }^{+} \mathrm{CCR} 7^{+} \mathrm{EM}$ ( $1.5 \%$ per day), and $\mathrm{CD}^{+}{ }^{+} \mathrm{CCR}^{-} \mathrm{CM}$ (4.7\% per day) $\mathrm{T}$ cells were estimated from these data (56). Because $57 \%$ of the memory cells were CM T cells, the average turnover rate of memory $\mathrm{CD} 4{ }^{+} \mathrm{T}$ cells was $3.3 \%$ per day, which is close to the earlier estimate (55) (Table 1). T-cell proliferation rates were found to be very similar in young and elderly people (57) and were in the same range as in the other two studies with this labeling regimen and mathematical model (Table 1). A possible caveat of these three studies (55-57) is that the earliest data point was taken at day 3, whereas labeling was stopped at day 1 . The fraction of labeled cells at the peak could therefore not be measured and was estimated by extrapolating the exponential loss of labeled cells during the down-labeling phase to day 1 . The estimated proliferation rates (and half-lives in Table 1) are quite sensitive to this extrapolation because the predicted exponential loss during the first days is relatively steep. If the true peak occurred somewhat later than day 1 , that is the time-point at which ${ }^{2} \mathrm{H}_{2}$-glucose was withdrawn, the proliferation rate $p$ may have been overestimated several folds.

The T-cell half-lives shown in Table 1 are all based on average proliferation rates that are largely estimated from the upslope during the up-labeling period. The downslopes during delabeling provide an estimate for the death rate of recently divided cells $(5,6,58)$, which tend to be larger than the average turnover rates that we are interested in. Although both uplabeling and down-labeling data are essential for estimating $P$ and $d^{*}$, most data sets take most of their samples during the delabeling phase only. There is only one published data set on deuterium labeling with sufficient sampling points during the up-labeling phase to reliably estimate the approach to the peak. Mohri et al. (7) labeled human volunteers for 1 week with ${ }^{2} \mathrm{H}_{2}$ glucose, drawing daily blood samples during the up-labeling phase. The percentages of labeled T-cells increased approximately linearly toward peak values of $2-3 \%$ at day 7 (7). We have refitted the data from the healthy controls (7) with the model proposed by Asquith et al. (6) (Eqn 10) and found very similar fits and proliferation rates $p$, as were originally estimated with the earlier source, proliferation, and death models (7). The estimated T-cell half-lives from this study (Table 1) would therefore remain the same if they had been estimated with the model of Eqn 10. This can be understood from the early upslopes of these mathematical models. At early time-points, Eqn 10 approaches $\mathrm{f}=\mathrm{pt}$, which is in good agreement with the observed linear increase of the percentage of labeled cells. Because the early upslope of the model by Mohri et al. (7) also approaches $\mathrm{f}=\mathrm{pt}$, the models were indeed expected to estimate very similar average turnover rates $p$.

Our refitting of the data by Mohri et al. (7) was giving larger death rates $\left(d^{*}\right)$ than the death rates (d) that were originally estimated (results not shown). This is also natural because the models differ markedly in the biological interpretation of this death rate. In the model proposed by Asquith et al. (6), d* is the death rate of labeled cells, which are not assumed to be in steady state. The other models make use of the fact that the total population size is not changing, and their death rate $d$ is satisfying the steady state of the population (7-9).

We very recently finished a long-term labeling study in five healthy humans, aged 20-25 years, using ${ }^{2} \mathrm{H}_{2} \mathrm{O}$. During a 9week up-labeling and subsequent 16-week down-labeling period, blood samples were drawn at 14 different time-points. By analyzing the enrichment of the naive and memory $\mathrm{CD} 4^{+}$ and $\mathrm{CD}^{+}{ }^{+}$-cell populations, using a mathematical model based on the model by Asquith et al. (6), we estimated the halflives of naive $\mathrm{CD}^{+}{ }^{+}$and $\mathrm{CD} 8^{+} \mathrm{T}$ cells to be as long as 4.2 and 6.6 years, respectively, whereas memory $\mathrm{CD}^{+}{ }^{+}$and $\mathrm{CD} 8^{+} \mathrm{T}$ cells had half-lives of 0.4 and 0.7 years, respectively (Vrisekoop et al., manuscript submitted) (Table 1).

Summarizing, labeling with stable isotopes provides an attractive alternative for the use of BrdU because stable isotopes are non-toxic and because changes in label enrichment after cessation of stable isotope administration truly represent the loss of labeled cells and not - as in the case of BrdU - the difference between cell loss and proliferation. The exact estimates of lymphocyte kinetics resulting from stable isotope labeling depend both on the method of labeling and on the specific model used (Table 1). First, parameter estimates are sensitive to the duration of label administration. The longer the period of label administration, the more representative the fraction of labeled cells is for the whole population (6), showing the advantage of the use of ${ }^{2} \mathrm{H}_{2} \mathrm{O}$, which has been administered orally for long periods of time, compared with ${ }^{2} \mathrm{H}_{2}$-glucose, which has typically been administered intravenously. This effect of the duration of label administration is indeed evident in the studies summarized in Table 1. Second, 
values based on the precursor-product relationship tend to underestimate lymphocyte turnover, that is give rise to longer half-lives, than estimates based on mathematical models in which proliferation and loss rates can be analyzed separately. Finally, parameter estimates made during label administration show less turnover than estimates that are derived during the decay phase because the turnover of cells that have recently divided tends to be higher than the average turnover in the total cell population. Interestingly, the common discrepancy between proliferation and loss rates in labeling data, which used to be a subject of concern, may in fact be taken to one's advantage. Under steady-state conditions, the loss rate of the $\mathrm{T}$ cell population under consideration will be close to the proliferation rate that is estimated during label administration. The rate at which labeled cells are lost, in contrast, provides information as to whether $\mathrm{T}$ cells that have divided tend to contribute to the T-cell population or tend to be lost.

\section{Conclusions}

The theoretical models reviewed here show how theoretical immunology has contributed to the quantification of lymphocyte dynamics and illustrate the pitfalls of mere intuitive interpretation of kinetic immunological data. Although telomere lengths are generally used to measure lymphocyte proliferation and TRECs are measured to quantify thymus output, mathematical models have shown that both telomere lengths and TREC data are strongly influenced by both lymphocyte proliferation and thymus output. Moreover, mathematical analysis of telomere data has pointed out that the parallel decline of naive and memory telomere lengths with age does not imply that naive and memory lymphocytes divide at equal rates. These insights have shown the limitations of the use of telomere lengths and TREC data in the quantification of lymphocyte dynamics.

Lymphocyte-labeling techniques, including BrdU and stable isotope labeling, are expected to provide better insights into lymphocyte dynamics. Even these data are difficult to interpret by intuition, however. Although BrdU up- and down-labeling are generally interpreted to reflect lymphocyte proliferation and death, respectively, mathematical models have pointed out that both the up- and the down-labeling phase are influenced by lymphocyte proliferation and death. This is not the case in stable-isotope-labeling experiments because lymphocyte proliferation in the absence of stable isotopes will never lead to the formation of new labeled DNA strands. In this respect, stableisotope-labeling data are more straightforward than BrdU data. However, even the interpretation of stable-isotope-labeling data hinges on the use of a good mathematical model. Unfortunately, many stable-isotope-labeling data have so far been analyzed using the precursor-product relationship, which as we have shown above may lead to false immunological interpretations.

There is only one published stable-isotope-labeling study that has sufficient data points during the up- and down-labeling phase to reliably estimate the average turnover rate and the rate of loss of labeled cells (7). Our refitting of these data with the model proposed by Asquith et al. (6) confirmed the original estimates of average proliferation rates of 0.004 and 0.003 / day, that is half-lives of 173 and 231 days, of $C D 4^{+}$and CD ${ }^{+}{ }^{+}$cells, respectively, in healthy humans. Our own recent work based on long-term labeling of the naive and memory T-cell subsets with ${ }^{2} \mathrm{H}_{2} \mathrm{O}$ showed cellular half-lives of approximately 1500 and 2400 days for $\mathrm{CD}^{+}$and $\mathrm{CD}^{+}$naive $\mathrm{T}$ cells, respectively, whereas memory $\mathrm{CD}^{+}$and $\mathrm{CD}^{+} \mathrm{T}$ cells had half-lives of approximately 150 and 250 days, respectively (Vrisekoop et al.) (Table 1). The previously estimated half-lives of naive $\mathrm{T}$ cells (Table 1) are short compared with the more reliable 200-day Tcell half-life reported by Mohri et al. (7) and our own labeling results of the naive $\mathrm{T}$-cell subset. These differences are probably because of the short labeling periods and lack of samples around the peak in most previous studies.

Investments in the development of useful mathematical models will remain required because the interpretation of many kinetic data is too complex to be interpreted by intuition alone. Indeed, mathematical modeling is 'no more - but no less - than a way of thinking clearly' (61). The differences between the studies reviewed in this article show that we should intensify our efforts in both collecting better kinetic data and developing better mathematical models, allowing for more reliable parameter estimates. The current intensive collaboration between experimental and theoretical immunologists should therefore be expanded and nurtured if we indeed want to move immunology to a more quantitative field of science.

\section{References}

1. Mohri H, Bonhoeffer S, Monard S, Perelson AS, Ho DD. Rapid turnover of T lymphocytes in SIV-infected rhesus macaques. Science 1998;279:1223-1227.
2. Hellerstein M, et al. Directly measured kinetics of circulating $\mathrm{T}$ lymphocytes in normal and HIV-1-infected humans. Nat Med 1999;5: 83-89
3. Gett AV, Hodgkin PD. A cellular calculus for signal integration by $\mathrm{T}$ cells. Nat Immunol 2000;1:239-244. 
4. Revy P, Sospedra M, Barbour B, Trautmann A. Functional antigen-independent synapses formed between $\mathrm{T}$ cells and dendritic cells. Nat Immunol 2001;2:925-931.

5. Asquith B, Bangham CR. An introduction to lymphocyte and viral dynamics: the power and limitations of mathematical analysis. Proc R Soc Lond B Biol Sci 2003;270:1651-1657.

6. Asquith B, Debacq C, Macallan DC, Willems L, Bangham CR. Lymphocyte kinetics: the interpretation of labelling data. Trends Immunol 2002;23:596-601.

7. Mohri $\mathrm{H}$, et al. Increased turnover of $\mathrm{T}$ lymphocytes in HIV-1 infection and its reduction by antiretroviral therapy. J Exp Med 2001;194:1277-1288.

8. Ribeiro RM, Mohri H, Ho DD, Perelson AS In vivo dynamics of $\mathrm{T}$ cell activation, proliferation, and death in HIV-1 infection: why are $\mathrm{CD} 4+$ but not $\mathrm{CD} 8+\mathrm{T}$ cells depleted? Proc Natl Acad Sci USA 2002;99: 15572-15577.

9. Ribeiro RM, Mohri H, Ho DD, Perelson AS. Modeling deuterated glucose labeling of T-lymphocytes. Bull Math Biol 2002;64: 385-405.

10. Deenick EK, Gett AV, Hodgkin PD. Stochastic model of $\mathrm{T}$ cell proliferation: a calculus revealing IL-2 regulation of precursor frequencies, cell cycle time, and survival. J Immunol 2003;1 70:4963-4972.

11. De Boer RJ, Mohri H, Ho DD, Perelson AS. Estimating average cellular turnover from BrdU measurements. Proc R Soc Lond B Biol Sci 2003;270:849-858.

12. De Boer RJ, Mohri H, Ho DD, Perelson AS. Turnover rates of B cells, T cells, and NK cells in simian immunodeficiency virus-infected and uninfected rhesus macaques. J Immunol 2003;1 70:2479-2487.

13. Ganusov VV, Pilyugin SS, De Boer RJ, MuraliKrishna K, Ahmed R, Antia R. Quantifying cell turnover using CFSE data. J Immunol Methods 2005;298:183-200.

14. De Boer RJ, Perelson AS. Estimating division and death rates from CFSE data. J Comp Appl Math 2005;184:140-164.

15. De Boer RJ. Estimating the role of thymic output in HIV infection. Curr Opin HIV AIDS 2006;1:16-21.

16. Harley CB, Futcher AB, Greider CW. Telomeres shorten during ageing of human fibroblasts. Nature 1990;345:458-460.

17. Blackburn EH. Structure and function of telomeres. Nature 1991;350:569-573.

18. Hayflick L. Why do we live so long? Geriatrics 1988;43:77-79.

19. Hayflick L. Antecedents of cell aging research. Exp Gerontol 1989;24:355-365.

20. Allsopp RC, et al. Telomere length predicts replicative capacity of human fibroblasts. Proc Natl Acad Sci USA 1992;89:10114-10118.
21. Pilyugin S, Mittler J, Antia R. Modeling T-cell proliferation: an investigation of the consequences of the Hayflick limit. J Theor Biol 1997;186:117-129.

22. Weng NP, Levine BL, June CH, Hodes RJ. Human naive and memory T lymphocytes differ in telomeric length and replicative potential. Proc Natl Acad Sci USA 1995;92:11091-11094.

23. Michie CA, McLean A, Alcock C, Beverley PC Lifespan of human lymphocyte subsets defined by CD 45 isoforms. Nature 1992;360:264-265.

24. McLean AR, Michie CA. In vivo estimates of division and death rates of human $\mathrm{T}$ lymphocytes. Proc Natl Acad Sci USA 1995;92:3707-3711.

25. De Boer RJ, Noest AJ. T cell renewal rates, telomerase, and telomere length shortening. J Immunol 1998;160:5832-5837.

26. Weng NP, Hathcock KS, Hodes RJ. Regulation of telomere length and telomerase in $\mathrm{T}$ and $\mathrm{B}$ cells: a mechanism for maintaining replicative potential. Immunity 1998;9:151-157.

27. Counter CM, Gupta J, Harley CB, Leber B, Bacchetti S. Telomerase activity in normal leukocytes and in hematologic malignancies. Blood 1995;85:2315-2320.

28. Mackall CL, et al. Age, thymopoiesis, and CD4+ T-lymphocyte regeneration after intensive chemotherapy. N Engl J Med 1995;332:143-149.

29. Mackall CL, Bare CV, Granger LA, Sharrow SO, Titus JA, Gress RE. Thymic-independent $\mathrm{T}$ cell regeneration occurs via antigen-driven expansion of peripheral $\mathrm{T}$ cells resulting in a repertoire that is limited in diversity and prone to skewing. J Immunol 1996;156:4609-4616.

30. Mackall CL, Gress RE. Pathways of T-cell regeneration in mice and humans: implications for bone marrow transplantation and immunotherapy. Immunol Rev 1997;157:61-72.

31. Mackall CL, Gress RE. Thymic aging and T-cell regeneration. Immunol Rev 1997; 160:91-102.

32. Hazenberg MD, et al. Establishment of the CD4+ T-cell pool in healthy children and untreated children infected with HIV-1. Blood 2004;104:3513-3519.

33. Kong F, Chen CH, Cooper MD. Thymic function can be accurately monitored by the level of recent $\mathrm{T}$ cell emigrants in the circulation. Immunity 1998;8:97-104.

34. Douek DC, et al. Changes in thymic function with age and during the treatment of HIV infection. Nature 1998;396:690-695.

35. Douek DC, et al. Assessment of thymic output in adults after haematopoietic stem-cell transplantation and prediction of T-cell reconstitution. Lancet 2000;355:1875-1881.
36. Hazenberg MD, Borghans JAM, De Boer RJ, Miedema F. Thymic output: a bad TREC record. Nat Immunol 2003;4:97-99.

37. Hazenberg MD, et al. Increased cell division but not thymic dysfunction rapidly affects the T-cell receptor excision circle content of the naive $\mathrm{T}$ cell population in HIV-1 infection. Nat Med 2000;6: 1036-1042.

38. Dutilh B, De Boer RJ. Decline in excision circles is no evidence for homeostatic renewal of naive $\mathrm{T}$ cells. J Theor Biol 2003;224:351-358.

39. Harris JM, et al. Multiparameter evaluation of human thymic function: interpretations and caveats. Clin Immunol 2005;115: 138-146.

40. Lewin SR, et al. Dynamics of T cells and TCR excision circles differ after treatment of acute and chronic HIV infection. J Immunol 2002;169:4657-4666.

41. Nobile M, et al. De novo T-cell generation in patients at different ages and stages of HIV-1 disease. Blood 2004; 104:470-477.

42. Lempicki RA, et al. Impact of HIV-1 infection and highly active antiretroviral therapy on the kinetics of $\mathrm{CD} 4+$ and $\mathrm{CD} 8+\mathrm{T}$ cell turnover in HIV-infected patients. Proc Natl Acad Sci USA 2000;97:13778-13783.

43. Kovacs JA, et al. Identification of dynamically distinct subpopulations of $\mathrm{T}$ lymphocytes that are differentially affected by HIV. J Exp Med 2001;194:1731-1741.

44. Kovacs JA, et al. Induction of prolonged survival of $\mathrm{CD} 4+\mathrm{T}$ lymphocytes by intermittent IL-2 therapy in HIV-infected patients. J Clin Invest 2005;115:2139-2148.

45. Di Mascio M, et al. Naive T-cell dynamics in human immunodeficiency virus type 1 infection: effects of highly active antiretroviral therapy provide insights into the mechanisms of naive T-cell depletion. J Virol 2006;80:2665-2674.

46. Rocha B, Penit C, Baron C, Vasseur F, Dautigny N, Freitas AA. Accumulation of bromodeoxyuridine-labeled cells in central and peripheral lymphoid organs: minimal estimates of production and turnover rates of mature lymphocytes. Eur J Immunol 1990;20:1697-1708.

47. Tough DF, Sprent J. Turnover of naive- and memory-phenotype T cells. J Exp Med 1994;179:1127-1135.

48. Bonhoeffer S, Mohri H, Ho D, Perelson AS. Quantification of cell turnover kinetics using 5-bromo-2' -deoxyuridine. J Immunol 2000;164:5049-5054.

49. Debacq C, et al. Increased cell proliferation, but not reduced cell death, induces lymphocytosis in bovine leukemia virus-infected sheep. Proc Natl Acad Sci USA 2002;99:10048-10053. 
50. Hellerstein MK, Neese RA. Mass isotopomer distribution analysis: a technique for measuring biosynthesis and turnover of polymers. Am J Physiol 1992;263: E988-E1001.

51. Neese RA, et al. Measurement in vivo of proliferation rates of slow turnover cells by 2H2O labeling of the deoxyribose moiety of DNA. Proc Natl Acad Sci USA 2002;99: 15345-15350.

52. Macallan DC, Fullerton CA, Neese RA, Haddock K, Park SS, Hellerstein MK. Measurement of cell proliferation by labeling of DNA with stable isotope-labeled glucose: studies in vitro, in animals, and in humans. Proc Natl Acad Sci USA 1998;95:708-713.
53. McCune JM, et al. Factors influencing T-cell turnover in HIV-1-seropositive patients. J Clin Invest 2000;105:R1-R8.

54. Hellerstein MK, et al. Subpopulations of long-lived and short-lived T cells in advanced HIV-1 infection. J Clin Invest 2003;112: 956-966.

55. Macallan DC, et al. Measurement and modeling of human T cell kinetics. Eur J Immunol 2003;33:2316-2326.

56. Macallan DC, et al. Rapid turnover of effectormemory CD4(+) T cells in healthy humans. J Exp Med 2004;200:255-260.

57. Wallace DL, et al. Direct measurement of $\mathrm{T}$ cell subset kinetics in vivo in elderly men and women. J Immunol 2004;173: 1787-1794.
58. Grossman Z, Herberman RB, Dimitrov DS. $\mathrm{T}$ cell turnover in SIV infection. Science 1999;284:555a-555b.

59. Sachsenberg N, et al. Turnover of CD4+ and CD8+ T lymphocytes in HIV-1 infection as measured by Ki-67 antigen. J Exp Med 1998;187:1295-1303.

60. Hazenberg MD, et al. T-cell division in human immunodeficiency virus (HIV)-1 infection is mainly due to immune activation: a longitudinal analysis in patients before and during highly active antiretroviral therapy (HAART). Blood 2000;95:249-255.

61. May RM. Uses and abuses of mathematics in biology. Science 2004;303:790-793. 